\title{
Changes of Th1/Th2 cytokines in immunocompetent patients with pulmonary cryptococcosis
}

\author{
J.L. Wang, S.Y. Li, Y.F. Luo, Y.P. Zhai and X.Q. Wei \\ State Key Laboratory of Respiratory Disease, \\ Guangzhou Institute of Respiratory Disease, \\ The First Affiliated Hospital of Guangzhou Medical University, \\ Guangzhou Medical University, Respiratory Medicine, \\ Guangzhou, Guangdong, China \\ Corresponding author: S.Y. Li \\ E-mail: shiyuelicn@163.com
}

Genet. Mol. Res. 12 (4): 5733-5742 (2013)

Received May 14, 2013

Accepted September 21, 2013

Published November 18, 2013

DOI http://dx.doi.org/10.4238/2013.November.18.22

\begin{abstract}
The aim of this study was to investigate the changes of Th1/Th2 cytokines in immunocompetent patients with pulmonary cryptococcosis (PC). Twenty immunocompetent patients with PC were identified by histopathological examination and were enrolled in the study along with the age- and gender-matched healthy controls. The serum concentrations of interferon- $\gamma$ (IFN- $\gamma$ ), interleukin-4 (IL4), and interleukin-12 (IL-12) were measured by enzyme-linked immunosorbent assay (ELISA). Peripheral blood mononuclear cells (PBMCs) in both groups were isolated and incubated with or without recombinant human IL-12 (rhIL-12) for $48 \mathrm{~h}$, and the concentrations of IFN- $\gamma$ and IL-4 in the supernatant were measured by ELISA. Serum IFN- $\gamma$ levels were greatly decreased in the patients compared with control groups $(\mathrm{P}<0.01)$, whereas no significant difference was observed in serum IL-4 and IL-12 levels. The concentrations of IFN- $\gamma$ and IL-4 in the supernatant of PBMCs without the stimulation of rhIL-
\end{abstract}


12 showed no differences between the two groups. Treatment with rhIL12 stimulated the release of IFN- $\gamma$, but not IL-4, into the supernatant of PBMCs in both groups, with a lower increase observed in the patients (4.3-fold) compared to that of controls $(7.9$-fold) $(\mathrm{P}<0.01)$. Serum IFN- $\gamma$ levels may be dampened in immunocompetent patients with PC with no significant changes in serum IL-4 and IL-12 levels. The deficiency in the response to IL-12 stimulation of Th1 cells may be one of the underlying mechanisms for the decline in serum IFN- $\gamma$ levels.

Key words: Pulmonary cryptococcosis; Interleukin-4; Interferon- $\gamma$; Interleukin-12; Peripheral blood mononuclear cells

\section{INTRODUCTION}

Pulmonary cryptococcosis (PC) is an opportunistic infection of the lungs caused by Cryptococcus neoformans or Cryptococcus gattii (Velagapudi et al., 2009). PC is generally believed to occur mostly in immunocompromised patients such as those with HIV/AIDS, recipients of organ transplantations, those with diabetes mellitus, and those undergoing longterm treatment with immunosuppressants. In recent years, reports of PC without recognizable immune defects have significantly increased. A study in the US conducted in 2008 revealed that up to $25 \%$ of the PC infectious cases occurred in patients without recognizable immune defects (Baddley et al., 2008). Shanghai Pulmonary Hospital in China reported that up to $53.95 \%$ of the 76 PC patients admitted between 2001 and 2009 had no comorbidity or immune dysfunction (Zhang et al., 2012). The dissemination of Cryptococcus can result in severe cryptococcal meningitis, which is a major threat to human health worldwide because it is very hard to treat.

The outcome of cryptococcosis depends on the immune status of the infected individual and on the expression of host cytokines generated in response to the pathogen. The host defense against infection with Cryptococcus mainly occurs through the T cell-mediated immunity (CMI) system. According to the production of cytokines, helper T cells (Th) can be divided into Th1 cells, to primarily produce interferon (IFN)- $\gamma$ and interleukin (IL)-2, and Th2 cells, to primarily produce IL-4 and IL-10. Most IL-12 is produced by host macrophages and dendritic cells in response to infectious cryptococcus. After it binds to the IL-12 receptor at the surface of natural killer (NK) cells and T-lymphocytes, it induces Th0 cells to differentiate into Th1 cells (D'Elios et al., 2011). In a host with PC, IFN- $\gamma$, a representative cytokine of Th1 cells (Abbas et al., 1996), can enhance MHC-II expression and promote antigen presentation and activation on macrophages to start the body's immune response (Kawakami et al., 1995). IFN- $\gamma$ can also induce the synthesis of enzymes that mediate breathing outbreak to increase the generation of antiseptic materials like nitric oxide so that the sterilization ability on macrophages is enhanced (Kawakami et al., 1997; Herring et al., 2002; Arora et al., 2011). The macrophages play a role in enhancing cellular immunity and in regulating the immune response chiefly via the IL-12/ IFN- $\gamma$ pathway (Hoag et al., 1997; Decken et al., 1998; Kawakami et al., 1999; Lehtonen et al., 2003). At the same time, IL-4 (Kawakami et al., 1997), a representative cytokine of Th2 cells (D'Elios et al., 2011), can promote the secretion of urea from the macrophages, which are selectively activated by IL-4 (Hardison et al., 2010). The increased secretion of urea can in turn facilitate cryptococcal proliferation in the macrophages. Both Th1 and Th2 cytokines thus 
play important roles in cryptococcus infection and its outcome by means of mutual regulation and mutual inhibition of their respective functions.

In AIDS-associated cryptococcal patients, studies have confirmed that the host immune balance from Th1 to Th2 leads to the loss of $\mathrm{CD}^{+} \mathrm{T}$ cells (Lortholary et al., 2005; Zheng et al., 2007), and the cytokine levels were changed compared with controls. In immunodeficient patients with PC, the Th1 cytokines, such as IFN- $\gamma$, were higher, but the Th2 cytokines were lower (Pietrella et al., 1999). However, there has been no report investigating changes of Th1/ Th2 cytokines in immunocompetent patients with PC. Previous studies of Th1/Th2 cytokines have nearly all been conducted in animals (Hoag et al., 1997; Decken et al., 1998; Hernandez et al., 2005; Wormley et al., 2005; Chen et al., 2008). Therefore, the roles and effects of Th1/ Th2 cytokines in immunocompetent PC patients were investigated in the present study and compared with healthy controls. These results might facilitate understanding of the status of the CMI, the potential mechanisms underlying the changes, and ultimately contribute to the prevention and treatment of the disease.

\section{MATERIAL AND METHODS}

\section{Patients and diagnostic criteria}

Twenty immunocompetent patients with PC (PC group) were enrolled in the present study, whose diagnoses were confirmed by histopathological examinations, between February 2011 and February 2012 at the First Affiliated Hospital to Guangzhou Medical College during their hospitalization. This study was conducted in accordance with the Declaration of Helsinki and with approval from the Ethics Committee of The First Affiliated Hospital of Guangzhou Medical University. Written informed consent was obtained from all participants. All patients met the diagnostic criterion (Yi et al., 2004; Lu et al., 2009): detection of Cryptococcus by cytological or histological identification in pulmonary resection specimens or in all kinds of lung tissues obtained by invasive puncture biopsy. Twenty age- and gender-matched volunteers were also enrolled as controls. All control subjects had come to our Medical Examination Center for a physical check-up during the same period and had no abnormalities in chest X-ray examinations.

The PC patients recruited all had negative determination of HIV antibodies, a history of good health, no basic diseases, and met the following exclusion criteria (Chang et al., 2006; The Chinese Medical Journal Editor Committee, 2006; Ye et al., 2012; Zhang et al., 2012): 1) peripheral absolute neutrophil count below $2.0 \times 10^{9} / \mathrm{L}$ or absolute lymphocyte count below $0.9 \mathrm{x}$ $10^{9} / \mathrm{L}, 2$ ) humoral immune parameters below the lower limits of the normal range (IgG 6-16, IgA 0.7-5.0, and $\operatorname{IgM~0.6-2.0~g/L),~3)~undergoing~or~having~undergone~immunosuppressant~therapy,~}$ 4) having undergone organ transplantation or presence of graft versus host disease, 5) continuous administration of steroid hormones for more than 3 weeks, and 6) chronic diseases (including diabetes mellitus, cancer, and rheumatoid immune diseases, etc.), long stay in the intensive care unit after surgery or trauma, long-term mechanical ventilation, an indwelling catheter in the body, all parenteral nutrition, and long-term therapy by broad-spectrum antibiotics.

\section{Serum samples}

Five milliliters peripheral venous blood was obtained from participants in both 
groups. The serum was obtained by low speed centrifugation at $2500 \mathrm{rpm}$ using a conventional desktop centrifuge (Eppendorf 5702; Germany), repacked, and stored at $-80^{\circ} \mathrm{C}$. Enzymelinked immunosorbent assay (ELISA) was used to determine the serum concentrations of IL-12, IFN- $\gamma$, and IL-4. The Human IL-4 ELISA kit, Human IFN- $\gamma$ ELISA kit, and Human IL-12p70 ELISA kit were obtained from Dakewe Biotech Company, China.

\section{Isolation and culture of peripheral blood mononuclear cells (PBMCs)}

Ten milliliters sterile peripheral heparinized venous blood was obtained from participants in both groups. After low speed centrifugation at $2500 \mathrm{rpm}$, the upper blood plasma was removed, followed by addition of lymphocyte isolation liquid into the lower cells. PBMCs isolated from the whole blood were washed twice in RPMI 1640 and resuspended in culture medium at a concentration of $1 \times 10^{6}$ cells $/ \mathrm{mL}$. Then, $0.5 \mathrm{~mL}$ cell suspension was added to the wells of a 24-well tissue culture plate. Next, an additional $0.5 \mathrm{~mL}$ RPMI 1640 was added into the wells in the control group while an additional $0.5 \mathrm{~mL} 20 \mathrm{ng} / \mathrm{mL}$ recombinant human (rh)IL-12 (ProSpec Biotech Company; USA) was added in the PC group. Plates were incubated for $48 \mathrm{~h}$ at $37^{\circ} \mathrm{C}$ and $5 \% \mathrm{CO}_{2}$. Culture supernatants were harvested and assayed for levels of IFN- $\gamma$ and IL-4 by ELISA.

\section{Statistical analysis}

All data are reported as means \pm standard error. The Student $t$-test was used to compare numerical data among groups. Significance was set to $\mathrm{P}<0.05$ for all statistical analyses.

\section{RESULTS}

\section{Basic data}

The 20 immunocompetent patients with PC (PC group) enrolled in the study were 16 males and 4 females, with an average age of $37.70 \pm 2.399$ years (range, 20 to 65 years). Among these patients, 4 had a history of smoking. Prior to developing PC, the patients were in good health and were free of basic diseases (Table 1). The 20 healthy volunteers (control group) were 15 males and 5 females, with an average age of $35.90 \pm 2.014$ years (range, 24 to 55 years). Of these patients, 2 had a history of smoking. These patients were also in good health and were free of basic diseases. There were no significant differences between the two groups in gender $(\mathrm{P}=1.0)$, age $(\mathrm{t}=0.5747, \mathrm{P}=0.5689)$, or history of smoking $(\mathrm{P}=0.661)$.

\section{Clinical signs and symptoms}

In this group, 6 patients $(30 \%)$ did not present any symptom, and their pulmonary diseases were detected by incidental radiological examination [chest X-ray or computed tomography (CT) scan]. The other 14 patients $(70 \%)$ had a variety of respiratory symptoms, including cough $(60 \%)$, fever $(20 \%)$, productive sputum $(15 \%)$, hemoptysis $(10 \%)$, and chest pain (5\%). There were no significantly positive signs in the chest physical examination (Table 1). 
Table 1. Demographic and clinical characteristics of immunocompetent patients with pulmonary cryptococcosis.

\begin{tabular}{lc}
\hline Variables $^{a}$ & All patients (N=20) \\
\hline Demographics & \\
Age (years) & $37.70 \pm 2.399$ \\
Gender & $4(20 \%)$ \\
Female & $16(80 \%)$ \\
Male & $4(20 \%)$ \\
Smoking & $0(\%)$ \\
Underlying diseases & $6(30 \%)$ \\
Symptoms & $12(60 \%)$ \\
No symptom & $4(20 \%)$ \\
Cough & $3(15 \%)$ \\
Fever & $2(10 \%)$ \\
Spit phlegm & $1(5 \%)$ \\
Hemoptysis & $9(45 \%)$ \\
Chest pain & $6(30 \%)$ \\
Diagnosis by & $5(25 \%)$ \\
VATS & \\
TBLB & \\
PCNB & \\
\hline
\end{tabular}

${ }^{a}$ Age is reported as means $\pm \mathrm{SE}$ and categorical data are presented as counts (percentages). VATS $=$ video-assisted thoracoscopic surgery; TBLB = transbronchial lung biopsy; PCNB = percutaneous cutting needle biopsy.

\section{Laboratory examinations}

The total counts and classifications of white blood cells and humoral immune parameters (including serum IgG, IgA, and IgM) were all normal in all PC patients.

\section{Radiological assessments}

All PC patients had a spiral CT scan on the chest. The main findings of the CT scans were classified into the 3 following patterns: 1 ) nodular ( 9 patients, $45 \%$ ), including 5 solitary nodular patients $(25 \%)$ (Figure 1) and 4 multi-nodular patients $(20 \%) ; 2)$ pneumonic $(6$ patients, 30\%) (Figure 2); 3) mixed morphological characteristics (nodular and pneumonic) ( 5 patients, $25 \%$ ). There were also cases of combined cavity (4 patients, $20 \%$ ), mediastinal lymphadenopathy ( 3 patients, $5 \%$ ), and pleural effusion ( 1 patient, $5 \%$ ).

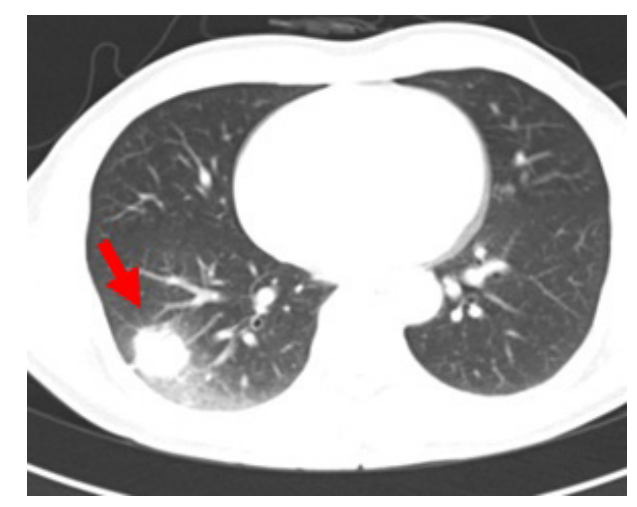

Figure 1. Solitary nodular revealed in the lung by chest CT scan. 


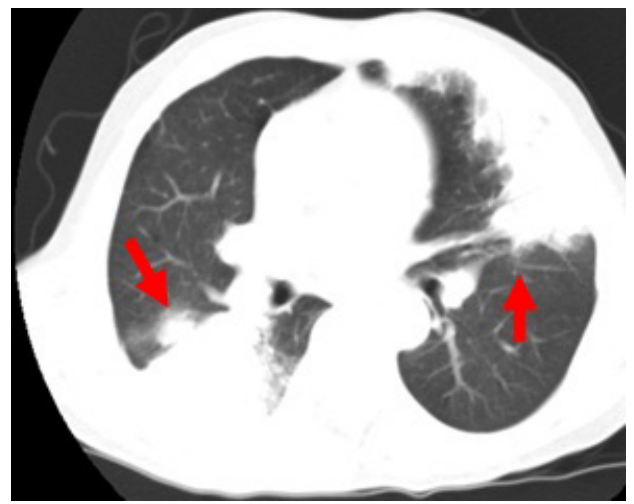

Figure 2. Multiple pneumonia revealed in the lung by chest CT scan.

\section{Diagnosis}

Cytological or histological identification of the 20 PC patients was confirmed by videoassisted thoracoscopic surgery ( 9 patients, $45 \%$ ), transbronchial lung biopsy ( 6 patients, $30 \%$ ), and percutaneous cutting needle biopsy ( 5 patients, 25\%). For pathological diagnosis, all specimens were managed with Gomori methenamine silver (GMS), periodic acid Schiff (PAS), and alcian blue $(\mathrm{AB})$ staining, and were examined by two experienced pathologists independently (Table 1). Twelve patients were GMS-positive, 13 were PAS-positive, and 6 were AB-positive.

\section{Average concentrations of serum IL-12, IFN- $\gamma$, and IL-4 in the two groups}

The average concentrations of serum IL-12, IFN- $\gamma$, and IL-4 in the PC group and in the control group were respectively $2.514 \pm 0.54 \mathrm{pg} / \mathrm{mL}$ vs $2.517 \pm 0.58 \mathrm{pg} / \mathrm{mL}, 14.52 \pm 2.71 \mathrm{pg} / \mathrm{mL}$ vs $81.80 \pm 9.84 \mathrm{pg} / \mathrm{mL}$, and $6.90 \pm 1.25 \mathrm{pg} / \mathrm{mL}$ vs $7.31 \pm 1.47 \mathrm{pg} / \mathrm{mL}$. The serum IFN- $\gamma$ levels were significantly lower in the PC group than in the control group $(\mathrm{P}<0.01)$. No significant differences in serum IL-4 and IL-12 levels were observed between the two groups (Figure 3A).
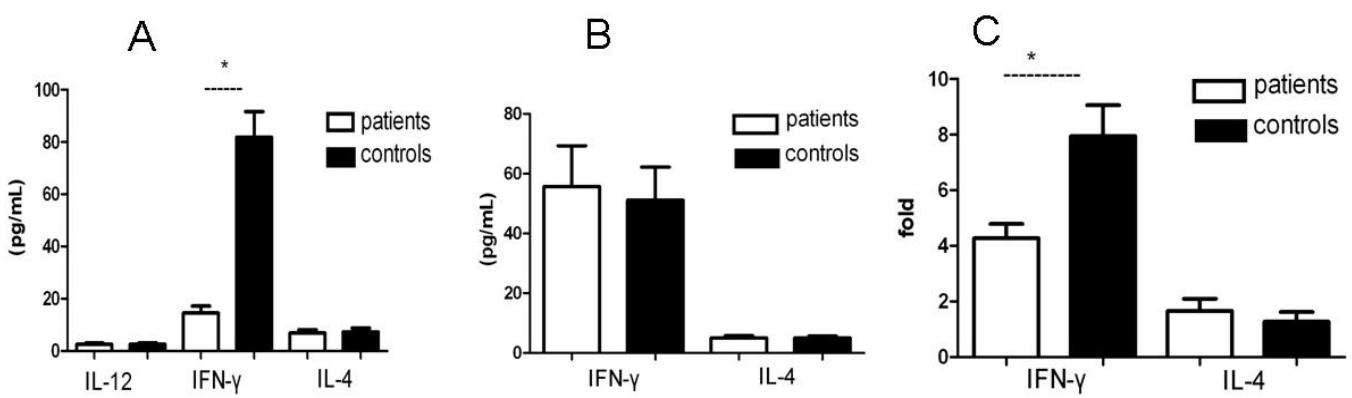

Figure 3. Concentrations of IL-12, IFN- $\gamma$, and in IL-4 in patient and in control groups. A. Serum IFN- $\gamma$ was greatly decreased in the patients $\left({ }^{*} \mathrm{P}<0.01\right)$. There were no significant differences in IL-4 and IL-12 levels between the 2 groups. B. Concentrations of IFN- $\gamma$ and IL- 4 in the supernatant of PBMCs without rhIL12 stimulation showed no differences between the two groups. C. Treatment with rhIL-12 stimulated the release of IFN- $\gamma$ but not IL-4 into the supernatant of PBMCs in both groups, with a lower increase observed in the patients $(4.3$-fold $)$ compared to the controls $(7.9$-fold $)(* \mathrm{P}<0.01)$. 


\section{IFN- $\gamma$ and IL-4 productions after stimulating PBMCs in culture with rhIL-12}

The average concentrations of supernatant IFN- $\gamma$ and IL-4 in the PC and control groups without rhIL-12 stimulation were respectively $55.69 \pm 13.61 \mathrm{pg} / \mathrm{mL}$ vs $51.13 \pm 17.54 \mathrm{pg} / \mathrm{mL}$ and $5.056 \pm 0.6785 \mathrm{pg} / \mathrm{mL} v s 5.025 \pm 0.6403 \mathrm{pg} / \mathrm{mL}$. There were no significant differences between the two groups regarding the above average concentrations (Figure 3B). rhIL-12 treatment increased the IFN- $\gamma$ levels in both groups, but the increment of change in PBMCs was much lower in the PC group than in the control group $(4.3 \pm 0.5$-fold $v s 7.9 \pm 1.1$-fold $)$ $(\mathrm{P}<0.01)$. There was no significant difference between the two groups regarding the IL-4 increment of change $(0.86 \pm 0.43$-fold vs $1.27 \pm 0.35$-fold) (Figure $3 \mathrm{C})$.

\section{DISCUSSION}

Although we believe that all of the patients enrolled in the present study were immunocompetent PC patients, there are currently no unified criteria to evaluate the immune function of PC patients. At present, the practical assessment is always a comprehensive consideration of the presence or absence of basic diseases, a history of using immunosuppressive drugs, humoral immune parameters, the total count and classification of white blood cells, serum $\mathrm{CD}^{+}$and $\mathrm{CD} 8^{+}$levels, etc. (Chang et al., 2006; The Chinese Medical Journal Editor Committee, 2006; Ye et al., 2012; Zhang et al., 2012). The patients enrolled in the study all used to be in good health, and had no basic diseases or a history of using immunosuppressants. They were diagnosed with PC by 2 experienced pathologists independently according to the results of their lung tissue examinations and by meeting the diagnostic criteria for PC (Yi et al., 2004; Lu et al., 2009). Immunocompetent PC patients can usually be mildly symptomatic or even asymptomatic. Up to $30 \%$ of the PC patients enrolled in the present study had no respiratory symptoms and their illness was incidentally detected in a regular physical checkup. The respiratory symptoms in the other PC patients included mild cough $(60 \%)$, low-grade fever $(20 \%)$, expectoration $(15 \%)$, hemoptysis $(10 \%)$, and chest pain $(5 \%)$, in agreement with what had been reported in the literature (Yi et al., 2004; Baddley et al., 2008; Lu et al., 2009; Zhang et al., 2012). The manifestations of PC on chest CTs can be diverse, but are mainly nodular, pneumonic, and mixed nodular and pneumonic characteristics. PC can also be concomitant with mediastinal lymphadenopathy, cavity, pleural effusion, etc. The nodular type was previously shown to be more common in immunocompetent patients (Zhang et al., 2012). In the present study, the PC patients of nodular type accounted for $45 \%$. Moreover, laboratory examinations showed that their white blood cells, neutrophils, lymphocytes, and serum immune globulins were all within the normal range. Therefore, they could be considered as immunocompetent.

Our results showed that serum IFN- $\gamma$ was significantly decreased in the PC group compared with the controls, whereas no significant difference regarding serum IL-4 was observed between the 2 groups. The reduction of IFN- $\gamma$ (Th1 cytokines) we observed is consistent with the findings of animal experiments (Wormley et al., 2005; Chen et al., 2008). In an animal experiment, Chen et al. (2008) reported that IFN- $\gamma$ production gradually reduced post-infection in pulmonary leukocyte culture supernatants in C. neoformans-infected mice. Wormley et al. (2005) reported that serum IL-4 increased, but that IFN- $\gamma$ decreased in $C$. neoformans-infected mice. Their conclusions indicated that Th1 cells in the C. neoformans-in- 
fected mice were immunologically deficient and susceptible to Cryptococcus. In HIV-infected individuals, research confirmed that HIV infection seems to be deficient of Th1 cells and shifts the host immune balance from a Th1- to a Th2-dominant state apart from leading to the loss of CD4 $4^{+} \mathrm{T}$ cells. HIV-infected individuals are susceptible to Cryptococcus and PC. Even cryptococcal meningitis can usually be found in AIDS patients (Zheng et al., 2007). Therefore, we believe that the significant decrease of serum IFN- $\gamma$ might be one cause of the susceptibility to Cryptococcus in PC patients. However, the serum IL-4 level (Th2 cytokines) we observed is inconsistent with previous reports (Hernandez et al., 2005; Wormley et al., 2005). The possible reason for this different finding in immunocompetent PC patients may be that the reaction of their competent immune systems to Cryptococcus leads to no enhancement in Th2 cells.

Our results also showed that concentrations of IFN- $\gamma$ and IL-4 in the supernatant of PBMC without rhIL-12 culture medium were similar in both $\mathrm{PC}$ and control groups $(\mathrm{P}>0.05)$. The rhIL-12 stimulation only resulted in a 4.3-fold increase of IFN- $\gamma$ levels in the supernatant of the PBMC culture medium, which was significantly lower than the 7.9-fold increase observed in the control group. This indicates that a mechanism may exist in the immunocompetent PC patients that may be responsible for the decreased secretion of IFN- $\gamma$ by Thl cells. With respect to the mechanisms of the immunity change, early in 1984, Blackstock and Hall (1984) found that immune suppression following cryptococcosis occurred as a result of infection with $C$. neoformans, and that the induction of adherent and non-adherent suppressor $\mathrm{T}$ cells in the spleen of the infected mice might be at least one of the possible mechanisms leading to the cellular immune function changed in cryptococcus-infected mice. Pericolini et al. (2010) concluded that galactoxylomannan (GalXM), the antigen composition of Cryptococcus, antagonized the activation of $\mathrm{T}$ cells and induced their death. This may be a mechanism responsible for the immune paralysis that follows GalXM administration, and it explains the powerful immunosuppression that accompanies cryptococcosis. Moreover, Szabo et al. (1997) found that antigens of intracellular pathogens downregulated the expression of the IL-12R $\beta 2$ subunit on the cellular surface of Th1 cells to cause reduced reactivity and sensitivity of Th1 cells to IL-12 cytokines. Consequently, an insufficient amount of the secretion of IFN- $\gamma$ could be induced to meet the body's responsive need for antigenic stimulation, resulting in a weakened defensive reaction. Therefore, deficient reactivity and sensitivity to the IL-12 stimulation of the Th1 cells may be one of underlying mechanisms for the serum IFN- $\gamma$ decline in immunocompetent patients with PC. However, more studies are needed to clarify whether the deficient reactivity and sensitivity to the IL-12 stimulation of Th1 cells are caused by cryptococcal infection.

NK cells also play an important role in cryptococcal infection. IL-12, by binding to the IL-12 receptor on the surface of NK cells, induces secretion of IFN- $\gamma$ by NK cells following cryptococcal infection (Kawakami et al., 2000). Since changes in serum cytokines, such as Th1/ Th2 are associated with a variety of complicated factors, further studies are urgently needed to elucidate the mechanisms underlying these changes. In addition, on the one hand, after cryptococcal infection, the host will activate his immune function to remove the Cryptococcus, whereas, on the other hand, the Cryptococcus will inhibit the activation, proliferation, and survival of T cells and disrupt the Th1/Th2 balance to ensure its survival in the body (Alvarez and Casadevall, 2006; Voelz et al., 2009). Although it remains to be explored whether the decreased activity of Th1 cells is caused by cryptococcal infection in immunocompetent PC patients, our findings of reduced serum IFN- $\gamma$ levels and decreased reactivity to IL-12 of 
Th1 cells can be promising indications for such patients. Recently, Chen et al. (2002) also demonstrated that IL-12 regulated the Th1/Th2 balance, through inducing IFN- $\gamma$ secretion and inhibiting IL-4 synthesis, to play a role of protective immunity for patients with Mycobacterium tuberculosis. Roilides et al. (1999) found that co-incubation of PBMCs and IL-12 resulted in enhanced inhibition of the activity of Aspergillus fumigatus. Jarvis et al. (2012) found that the addition of short-course IFN- $\gamma$ treatment to standard treatment significantly increased the rate of clearance of cryptococcal infection from the cerebrospinal fluid, and was not associated with any increase in adverse events in a randomized controlled trial. Research using IFN- $\gamma$ or rhIL12 for adjuvant therapy for such patients can be executed in the future.

In conclusion, we found that the serum IFN- $\gamma$ levels may be greatly reduced in immunocompetent PC patients, but that their serum IL-4 and IL-12 levels may not change obviously. Such patients present with deficiency in the response to IL-12 stimulation of Th1 cells, which may be one of the underlying mechanisms for the decline in serum IFN- $\gamma$ levels. These findings can serve as useful clinical indications for such patients. The therapeutic efficacy of pharmaceutics of IFN- $\gamma$ or rhIL-12, however, should be further observed and investigated in immunocompetent patients with PC.

\section{Conflicts of interest}

The authors declare that they have no conflict of interest.

\section{ACKNOWLEDGMENTS}

We thank Yaxia Tan and Guangqiao Zeng for their assistance with proofreading the manuscript.

\section{REFERENCES}

Abbas AK, Murphy KM, Sher A and Kenneth M (1996). Functional diversity of helper T lymphocytes. Nature 383: 787-793.

Alvarez M and Casadevall A (2006). Phagosome extrusion and host-cell survival after Cryptococcus neoformans phagocytosis by macrophages. Curr. Biol. 16: 2161-2165.

Arora S, Olszewski MA, Tsang TM, McDonald RA, et al. (2011). Effect of cytokine interplay on macrophage polarization during chronic pulmonary infection with Cryptococcus neoformans. Infect. Immun. 79: 1915-1926.

Baddley JW, Perfect JR, Oster RA, Larsen RA, et al. (2008). Pulmonary cryptococcosis in patients without HIV infection: factors associated with disseminated disease. Eur. J. Clin. Microbiol. Infect. Dis. 27: 937-943.

Blackstock R and Hall NK (1984). Non-specific immunosuppression by Cryptococcus neoformans infection. Mycopathologia 86: 35-43.

Chang WC, Tzao C, Hsu HH, Lee SC, et al. (2006). Pulmonary cryptococcosis: comparison of clinical and radiographic characteristics in immunocompetent and immunocompromised patients. Chest 129: 333-340.

Chen GH, McNamara DA, Hernandez Y, Huffnagle GB, et al. (2008). Inheritance of immune polarization patterns is linked to resistance versus susceptibility to Cryptococcus neoformans in a mouse model. Infect. Immun. 76: 2379-2391.

Chen J, Dong D, Yang Y, Sun X, et al. (2002). The effect of interleukin-12 on the production of Th(1) and Th(2) cytokines by peripheral blood mononuclear cells from patients with tuberculosis. Zhonghua Jie He He Hu Xi Za Zhi. 25: 292-295.

D'Elios MM, Benagiano M, della Bella C and Amedei A (2011). T-cell response to bacterial agents. J. Infect. Develop. Ctries. 5: 640-645.

Decken K, Kohler G, Palmer-Lehmann K, Wunderlin A, et al. (1998). Interleukin-12 is essential for a protective Th1 response in mice infected with Cryptococcus neoformans. Infect. Immun. 66: 4994-5000.

Hardison SE, Ravi S, Wozniak KL, Young ML, et al. (2010). Pulmonary infection with an interferon-gamma-producing Cryptococcus neoformans strain results in classical macrophage activation and protection. Am. J. Pathol. 176: 774-785. 
Hernandez Y, Arora S, Erb-Downward JR, McDonald RA, et al. (2005). Distinct roles for IL-4 and IL-10 in regulating T2 immunity during allergic bronchopulmonary mycosis. J. Immunol. 174: 1027-1036.

Herring AC, Lee J, McDonald RA, Toews GB, et al. (2002). Induction of interleukin-12 and gamma interferon requires tumor necrosis factor alpha for protective T1-cell-mediated immunity to pulmonary Cryptococcus neoformans infection. Infect. Immun. 70: 2959-2964.

Hoag KA, Lipscomb MF, Izzo AA and Street NE (1997). IL-12 and IFN-gamma are required for initiating the protective Th1 response to pulmonary cryptococcosis in resistant C.B-17 mice. Am. J. Respir. Cell Mol. Biol. 17: 733-739.

Jarvis JN, Meintjes G, Rebe K, Williams GN, et al. (2012). Adjunctive interferon-gamma immunotherapy for the treatment of HIV-associated cryptococcal meningitis: a randomized controlled trial. AIDS 26: 1105-1113.

Kawakami K, Kohno S, Kadota J, Tohyama M, et al. (1995). T cell-dependent activation of macrophages and enhancement of their phagocytic activity in the lungs of mice inoculated with heat-killed Cryptococcus neoformans: involvement of IFN-gamma and its protective effect against cryptococcal infection. Microbiol. Immunol. 39: 135-143.

Kawakami K, Tohyama M, Qifeng X and Saito A (1997). Expression of cytokines and inducible nitric oxide synthase mRNA in the lungs of mice infected with Cryptococcus neoformans effects of interleukin-12. Infect. Immun. 65: $1307-1312$.

Kawakami K, Qureshi MH, Zhang T, Koguchi Y, et al. (1999). Interferon-gamma (IFN- $\gamma$ )-dependent protection and synthesis of chemoattractants for mononuclear leucocytes caused by IL-12 in the lungs of mice infected with Cryptococcus neoformans. Clin. Exp. Immunol. 117: 113-122.

Kawakami K, Koguchi Y, Qureshi MH, Yara S, et al. (2000). NK cells eliminate Cryptococcus neoformans by potentiating the fungicidal activity of macrophages rather than by directly killing them upon stimulation with IL-12 and IL-18. Microbiol. Immunol. 44: 1043-1050.

Lehtonen A, Lund R, Lahesmaa R, Julkunen I, et al. (2003). IFN-alpha and IL-12 activate IFN regulatory factor 1 (IRF-1), IRF-4, and IRF-8 gene expression in human NK and T cells. Cytokine 24: 81-90.

Lortholary O, Sitbon K and Dromer F (2005). Evidence for human immunodeficiency virus and Cryptococcus neoformans interactions in the pro-inflammatory and anti-inflammatory responses in blood during AIDS-associated cryptococcosis. Clin. Microbiol. Infect. 11: 296-300.

Lu SH, Hou YY, Tan YS, Liu T, et al. (2009). Clinicopathological analysis of primary pulmonary cryptococcosis. Zhonghua Jie He He Hu Xi Za Zhi 32: 430-433.

Pericolini E, Gabrielli E, Bistoni G, Cenci E, et al. (2010). Role of CD45 signaling pathway in galactoxylomannan-induced T cell damage. PLoS One 5: e12720.

Pietrella D, Monari C, Retini C, Palazzetti B, et al. (1999). HIV type 1 envelope glycoprotein gp120 induces development of a T helper type 2 response to Cryptococcus neoformans. AIDS 13: 2197-2207.

Roilides E, Tsaparidou S, Kadiltsoglou I, Sein T, et al. (1999). Interleukin-12 enhances antifungal activity of human mononuclear phagocytes against Aspergillus fumigatus: implications for a gamma interferon-independent pathway. Infect. Immun. 67: 3047-3050.

Szabo SJ, Dighe AS, Gubler U and Murphy KM (1997). Regulation of the interleukin (IL)-12R beta 2 subunit expression in developing T helper 1 (Th1) and Th2 cells. J. Exp. Med. 185: 817-824.

The Chinese Medical Journal Editor Committee (2006). Diagnostic standard and treatment principle of invasive pulmonary fungus infection (draft). Chin. J. Intern. Med. 45: 697-700.

Velagapudi R, Hsueh YP, Geunes-Boyer S, Wright JR, et al. (2009). Spores as infectious propagules of Cryptococcus neoformans. Infect. Immun. 77: 4345-4355.

Voelz K, Lammas DA and May RC (2009). Cytokine signaling regulates the outcome of intracellular macrophage parasitism by Cryptococcus neoformans. Infect. Immun. 77: 3450-3457.

Wormley FL Jr., Cox GM and Perfect JR (2005). Evaluation of host immune responses to pulmonary cryptococcosis using a temperature-sensitive C. neoformans calcineurin A mutant strain. Microb. Pathog. 38: 113-123.

Ye F, Xie JX, Zeng QS, Chen GQ, et al. (2012). Retrospective analysis of 76 immunocompetent patients with primary pulmonary cryptococcosis. Lung 190: 339-346.

Yi XH, Kong J, Zhu MF, Zhang Y, et al. (2004). Pathological diagnosis and ultrastructure features of primary pulmonary cryptococcosis: a study of 27 cases. Zhonghua Bing Li Xue Za Zhi. 33: 424-428.

Zhang Y, Li N, Zhang Y, Li H, et al. (2012). Clinical analysis of 76 patients pathologically diagnosed with pulmonary cryptococcosis. Eur. Respir. J. 40: 1191-1200.

Zheng CF, Ma LL, Jones GJ, Gill MJ, et al. (2007). Cytotoxic CD4+ T cells use granulysin to kill Cryptococcus neoformans, and activation of this pathway is defective in HIV patients. Blood 109: 2049-2057. 